\title{
The effectiveness of BNT162b2 mRNA vaccine against COVID-19 caused by Delta variant of SARS-CoV-2: a systematic review and meta-analysis
}

\author{
Chia Siang Chia Kow ${ }^{1,2}$ (D) Dinesh Sangarran Ramachandram² $\cdot$ Syed Shahzad Hasan ${ }^{3,4}$
}

Received: 28 September 2021 / Accepted: 20 December 2021 / Published online: 31 January 2022

(c) The Author(s), under exclusive licence to Springer Nature Switzerland AG 2022

\begin{abstract}
Meta-analyses were utilized to determine the overall effectiveness of BNT162b2 mRNA vaccine (Pfizer vaccine) against COVID-19 caused by Delta variant from large real-world studies. A systematic literature search with no language restriction was performed in electronic databases to identify eligible observational studies that reported the effectiveness of the BNT162b2 mRNA vaccine to prevent reverse transcription-polymerase chain reaction (RT-PCR) confirmed COVID-19 caused by Delta variant of SARS-CoV-2 (B.1.617.2). Random-effects meta-analysis model was used to estimate the pooled odds ratio (OR) at a 95\% confidence interval, and the vaccine effectiveness was indicated as (pooled OR - 1)/OR. Seven studies were included for this meta-analysis. The meta-analysis revealed that the administration of BNT162b2 mRNA vaccine protected against RT-PCR confirmed COVID-19 caused by Delta variant $\geq 21$ days after the first dose, with vaccine effectiveness of 55\% (95\% confidence interval 46-63\%), as well as $\geq 14$ days after the second dose, with vaccine effectiveness of $81 \%$ (95\% confidence interval 69-88\%). In conclusion, the BNT162b2 mRNA vaccine offers a substantial protection rate against RT-PCR confirmed COVID-19 caused by the Delta variant upon full vaccination, albeit with slightly reduced effectiveness relative to other strains of SARS-CoV-2.
\end{abstract}

Keywords BNT162b2 $\cdot$ COVID-19 $\cdot$ Delta $\cdot$ Vaccine $\cdot$ Variant

\section{Introduction}

The Delta variant of SARS-CoV-2, also known as B.1.617.2, belongs to a viral lineage of SARS-CoV-2 first identified in India during an intense wave of coronavirus disease 2019 (COVID-19) in April and May 2021. The Delta variant is highly transmissible, where it was reported that it could be more than twice as transmissible as the original strain of SARS-CoV-2 (Andrews et al. 2021). COVID-19 caused by Delta variant still lead to typical symptoms including headache, sore throat, runny nose, and fever, but cough and loss of smell are less common. The lineage has since proliferated and linked to a resurgence of COVID-19 cases in many parts of the world, including those with robust vaccination drives, and this may lead to phenomenon of hyperlocal outbreaks (concentrated amounts of cases in neighborhoods with low vaccination rate) which could overwhelm the healthcare 
system due to unequal proportion of vaccination across different areas (Blanquart et al. 2021). Therefore, there have been concerns in the medical fraternity that the currently available COVID-19 vaccines may not be adequate to protect against COVID-19 caused by the Delta variant (Bian et al. 2021). This paper aimed to summarize through metaanalyses the overall effectiveness of the BNT162b2 mRNA vaccine against COVID-19 caused by Delta variant from real-world studies.

\section{Methods}

This study was conducted and reported according to the recommendations outlined in the Preferred Reporting Items for Systematic Reviews and Meta-Analyses (PRISMA) guidelines (Page et al. 2021). Two investigators (CSK and SSH) independently conducted systematic literature search in several electronic databases, including PubMed, Google Scholar, Scopus, Web of Science, and medRxiv, in September 2021. The search strategy was designed to identify all publications which reported the effectiveness of the BNT162b2 mRNA vaccine to prevent reverse transcription-polymerase chain reaction (RT-PCR) confirmed COVID-19 caused by Delta variant of SARSCoV-2 (B.1.617.2). We applied various combinations of Boolean operators for the following keywords during our search: [(SARS-Cov-2 OR 2019-nCOv OR COVID-19 OR coronavirus) AND (vaccine or vaccination) AND (variant)]. In addition, the references from narrative reviews or other systematic reviews were cross-checked to identify additional missing publications during the initial search.

Studies were eligible for inclusion in our systematic review and meta-analysis if they (1) were observational studies (of any design, for example, case-control, cohort, case series); (2) reported the effectiveness of the BNT162b2 mRNA vaccine to prevent reverse transcription-polymerase chain reaction (RT-PCR) confirmed COVID-19 caused by Delta variant of SARS-CoV-2 (B.1.617.2); (3) compared vaccine effectiveness between vaccinated and unvaccinated individuals or between preand post-vaccination; and (4) reported adjusted effectiveness estimates. For two or more studies that utilized the same data source for their investigations on vaccine effectiveness, we included the study that performed analysis with the latest data cut-off date. Studies that utilized surrogate measures of vaccine effectiveness against COVID19 caused by Delta variant of SARS-CoV-2 by reporting vaccine effectiveness during Delta predominance period were excluded. Studies that reported unadjusted effectiveness estimates, and studies that reported the effectiveness of the vaccine to prevent COVID-19-related mortality or COVID-19-related hospitalization were also excluded. We did not include preprints editorials, commentaries, and narrative reviews.

The outcome of interest, namely vaccine effectiveness, was defined as a relative risk reduction in RT-PCR confirmed COVID-19 caused by Delta variant in vaccinated individuals (post-vaccination) compared with unvaccinated individuals (pre-vaccination) (Weinberg and Szilagyi 2010). All relevant information from the eligible studies was extracted and recorded in a pre-determined data collection table. The following information was extracted from each included study: first author's surname, year of publication, study design, country where the study was performed, number of participants, the incidence/ frequency of COVID-19 in both vaccinated and unvaccinated individuals, adjusted effectiveness estimates, and covariates adjusted in the study. Newcastle-Ottawa Scale was used for critical appraisal of the quality of included observational studies. Two investigators (CSK and SSH) independently evaluated the quality of studies with the Newcastle-Ottawa Scale (Wells et al. 2013) and a Newcastle-Ottawa Scale of at least 8 , indicating high quality. Consensus discussions between the two investigators were carried out to resolve disagreements on the inclusion of studies, extraction of study characteristics, and quality appraisal of included studies.

A random-effects model was used to estimate the pooled odds ratio (OR) for the occurrence of COVID-19 caused by Delta variant between vaccinated and unvaccinated individuals, at $95 \%$ confidence intervals, when three or more studies were reporting the same type of effect measure (either odds ratio or hazard ratio [HR]). We examined the heterogeneity between studies using the $I^{2}$ statistics and the $\chi^{2}$ test, with $50 \%$ and $p<0.10$, respectively, were considered as an indication of the presence of heterogeneity. The vaccine effectiveness was indicated as (pooled HR -1 )/HR or (pooled OR -1$) / O R$, together with a $95 \%$ confidence interval. All analyses were performed using Meta XL, version 5.3 (EpiGear International, Queensland, Australia).

\section{Results and discussion}

Our literature search yielded 4441 records. After deduplication and application of eligibility criteria, 14 relevant articles were shortlisted for inclusion through full-text examination (Fig. 1). Of these, eight studies were excluded since they utilized surrogate measures of vaccine effectiveness against COVID-19 caused by Delta variant of SARS-CoV-2 by reporting vaccine effectiveness during Delta predominance period, reporting the effectiveness of vaccines other than vaccines BNT162b2 mRNA vaccine, or reported unadjusted effectiveness estimates. Eventually, seven studies (Andrews et al. 2021; Martínez-Baz et al. 2021; Nasreen et al. 2021; 
Fig. 1 PRISMA flow diagram for study selection
Identification of studies via databases and registers

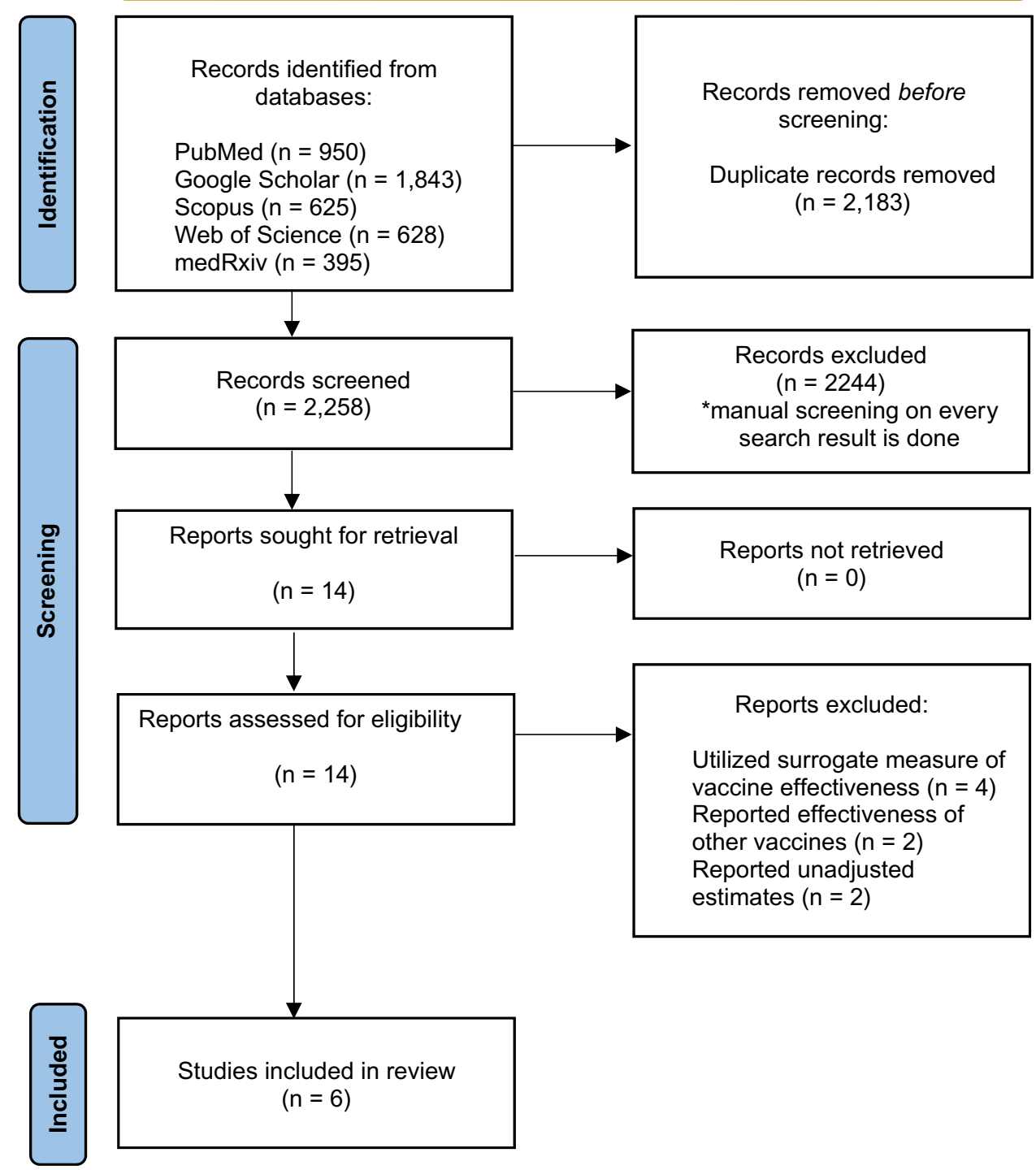

Sheikh et al. 2021; Skowronski et al. 2021; Tang et al. 2021; Tartof et al. 2021) were included in this systematic review and meta-analysis; all included studies were of retrospective design, with five case-control studies (Andrews et al. 2021; Nasreen et al. 2021; Sheikh et al. 2021; Skowronski et al. 2021; Tang et al. 2021) and two cohort studies (MartínezBaz et al. 2021; Tartof et al. 2021). The study characteristics are depicted in Table 1. The included studies were originated from Scotland (Sheikh et al. 2021), England (Andrews et al. 2021), Qatar (Tang et al. 2021), Canada (Nasreen et al. 2021; Skowronski et al. 2021) ( $n=2)$, Norway (Martínez-Baz et al. 2021), and the United States (Tartof et al. 2021). Age and sex were the most commonly adjusted covariates (adjusted in all included studies). Studies included for meta-analyses (Andrews et al. 2021; Martínez-Baz et al. 2021; Nasreen et al. 2021; Sheikh et al. 2021; Skowronski et al. 2021;
Tang et al. 2021) are deemed moderate-to-high quality with a Newcastle-Ottawa Scale ranging from 7 to 8 (Table 1).

The meta-analysis performed using the data extracted from three studies (Martínez-Baz et al. 2021; Nasreen et al. 2021; Tang et al. 2021) revealed a significant protective effect produced by the first dose of BNT162b2 mRNA vaccine (after 14 days or more) against SAR-CoV-2 infection caused by the Delta variant (pooled OR 0.42; 95\% confidence interval $0.36-0.49 ; I^{2}=0 \% ; p=0.63$; Fig. 2). The pooled estimate shows vaccine effectiveness of $58 \%(95 \%$ confidence interval 51-64\%). Similarly, the meta-analysis of two studies (Andrews et al. 2021; Nasreen et al. 2021) revealed a significant protective effect against SAR-CoV-2 infection caused by the Delta variant 21 days post first dose of BNT162b2 mRNA vaccine (pooled OR 0.45; $95 \%$ confidence interval $0.37-0.54 ; I^{2}=37 \% ; p=0.17$; Fig. 2 ), 


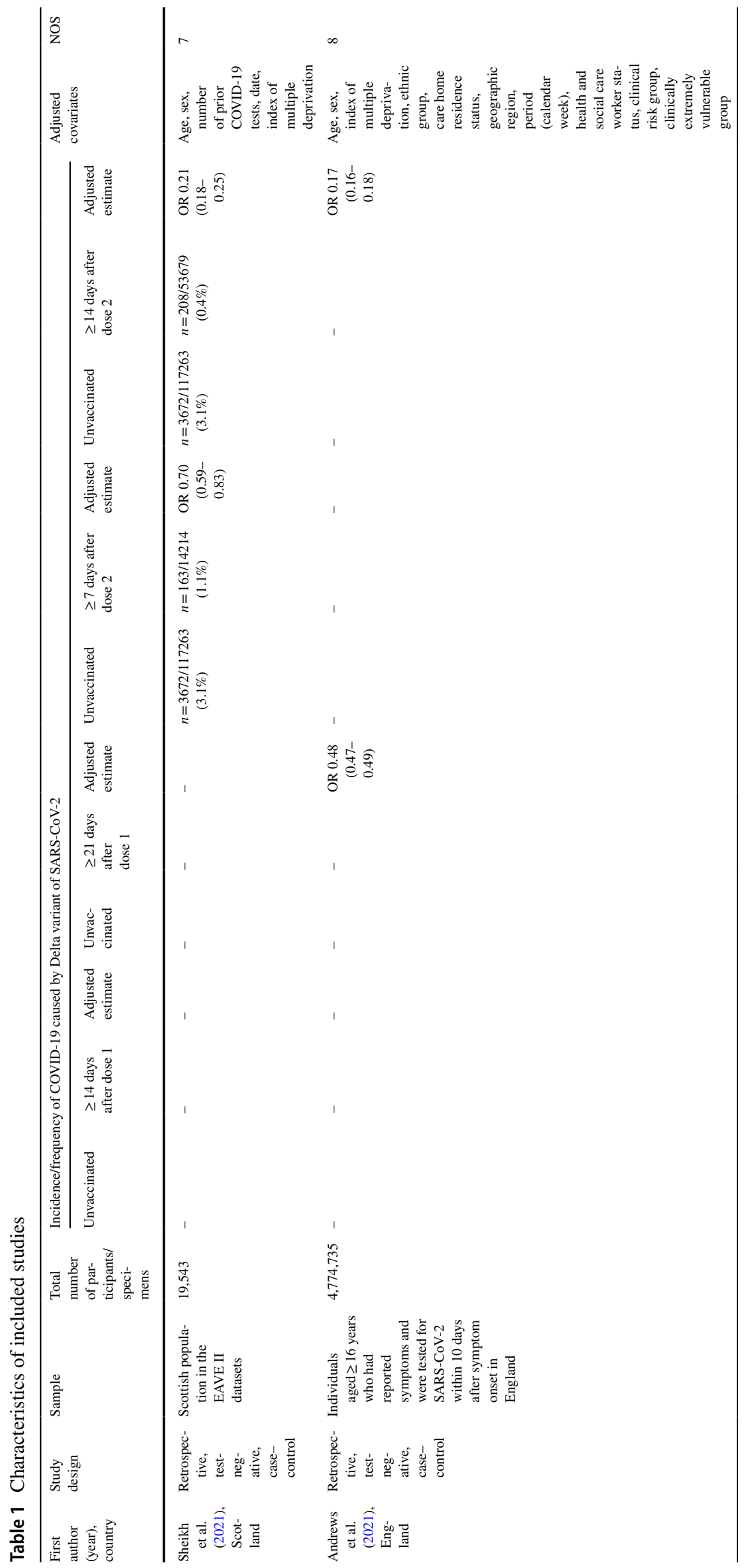




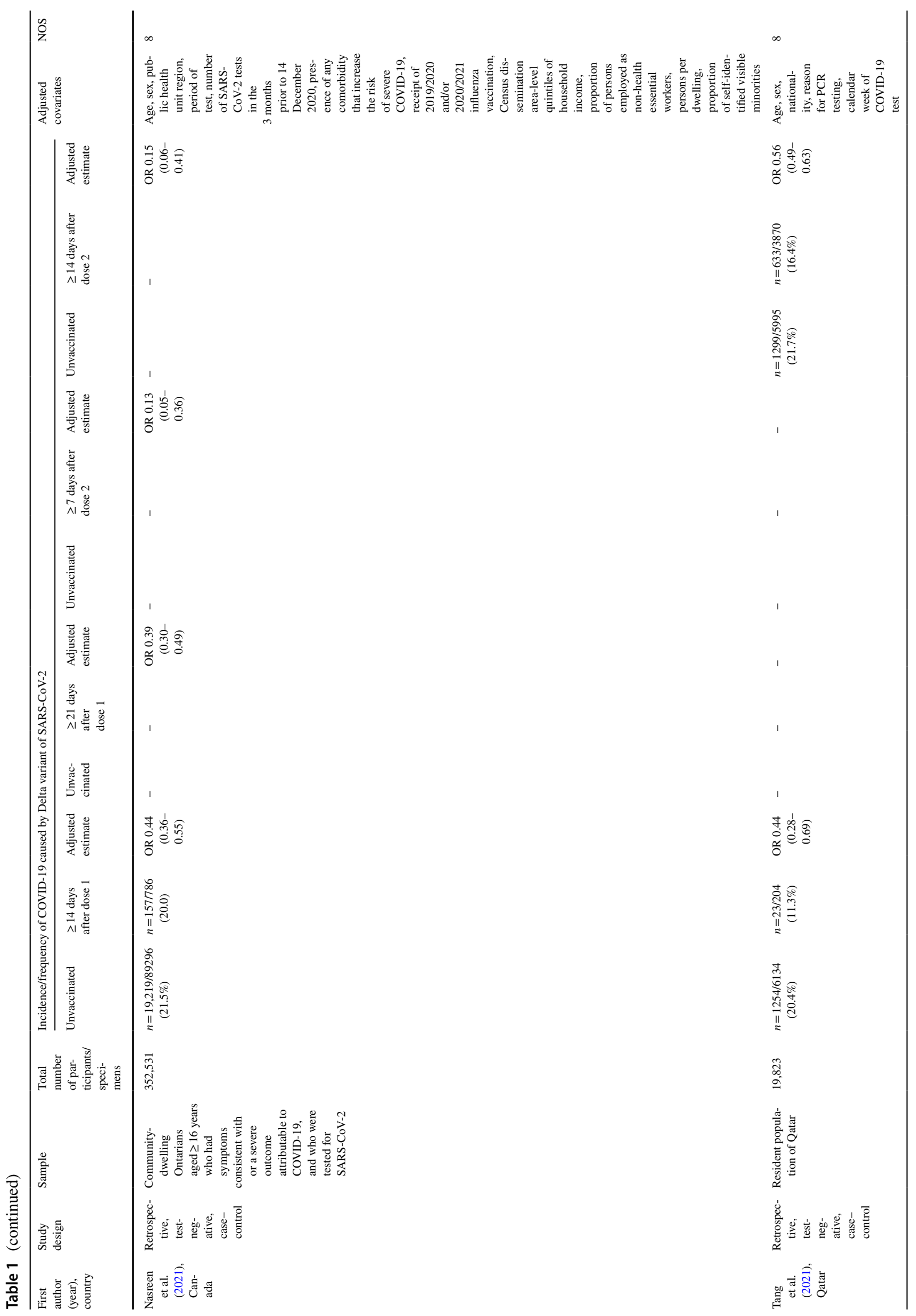




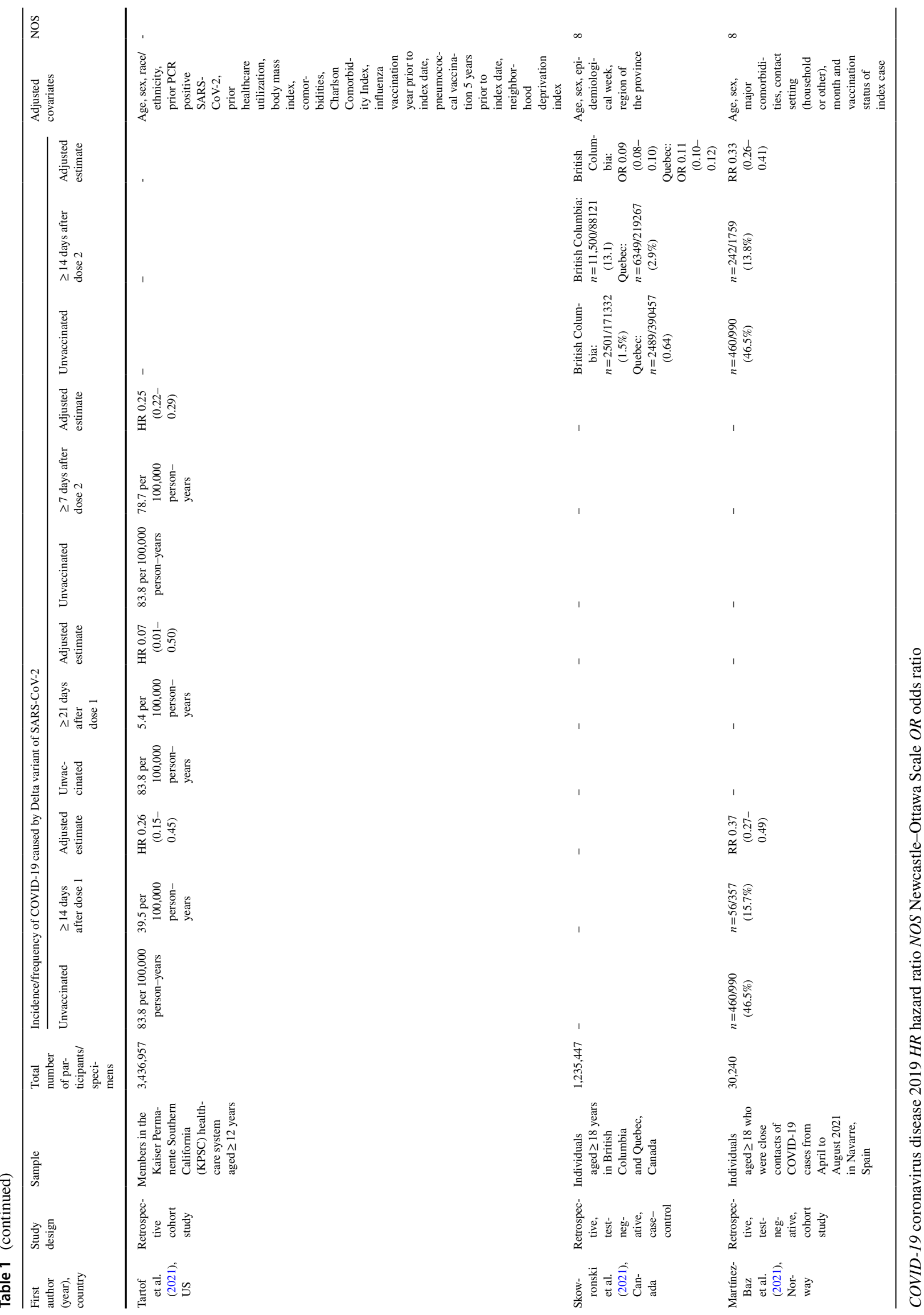




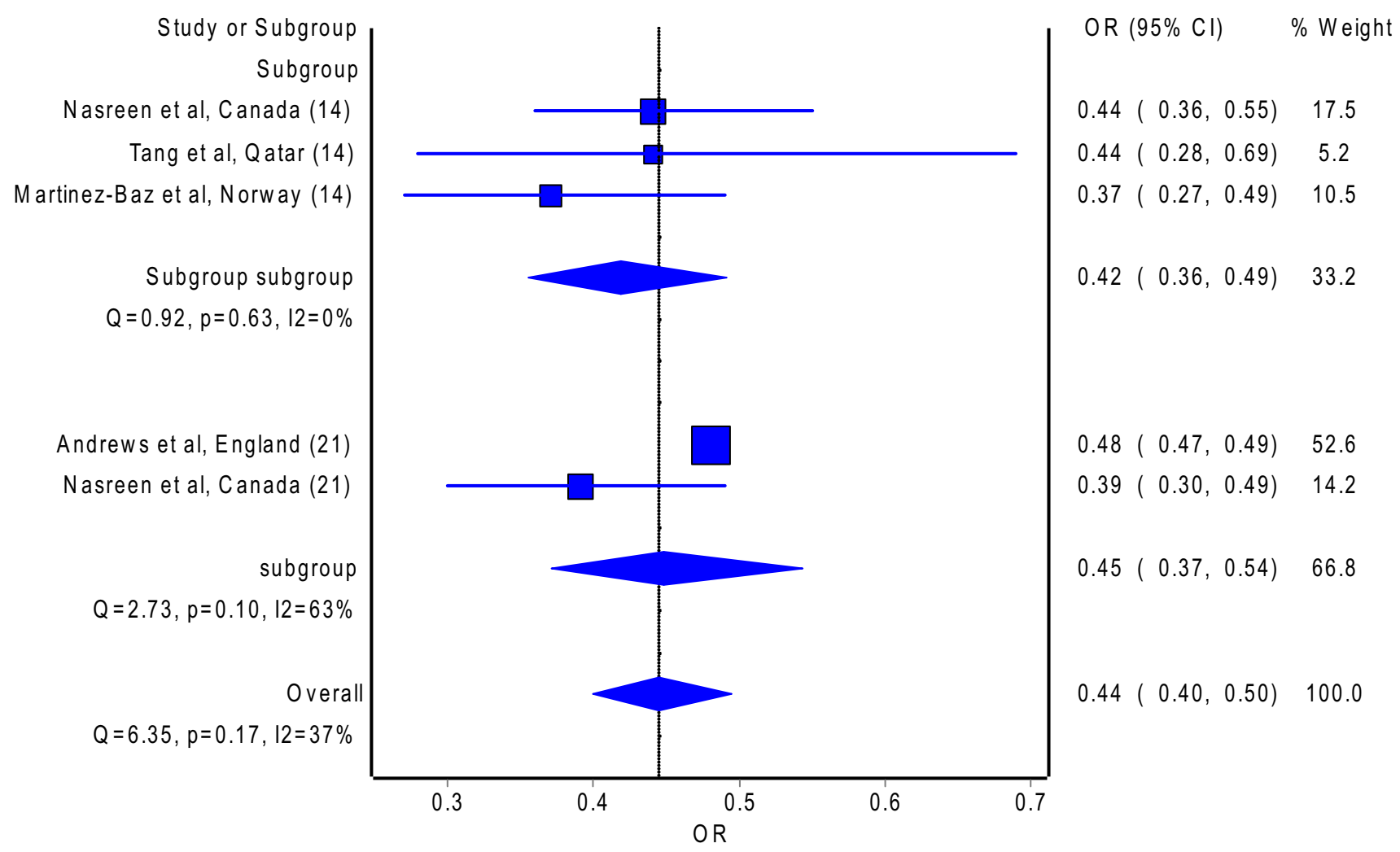

Fig. 2 Pooled odds ratio (OR) of the incidence of COVID-19 14 or 21 days post the first dose of vaccine relative to no vaccination

with vaccine effectiveness of 55\% (95\% confidence interval 46-63\%).

With the second dose of the BNT162b2 mRNA vaccine, our meta-analysis of six studies (Andrews et al. 2021; Martínez-Baz et al. 2021; Nasreen et al. 2021; Sheikh et al. 2021; Skowronski et al. 2021; Tang et al. 2021) documented an even higher significant protective effect measured at 14 days or more post second dose (pooled OR $0.19 ; 95 \%$ confidence interval $0.12-0.31 ; I^{2}=97 \% ; p=0.01$; Fig. 3 ), where the pooled estimate shows vaccine effectiveness of $81 \%$ (95\% confidence interval $69-88 \%$ ). Thus, there is adequate evidence against our model hypothesis of 'no significant protective effect' against SAR-CoV-2 infection caused by the Delta variant, at the current sample size.

Based on the findings, it appears that the BNT162b2 mRNA vaccine still offers substantial protection against RTPCR confirmed COVID-19 caused by the Delta variant in the real-world settings, in which partial vaccination (21 days or more after the first dose) reduced the risk of acquisition of COVID-19 caused by the Delta variant by 55\%, while full vaccination (14 days or more after the second dose) reduced the risk of acquisition of COVID-19 caused by the
Delta variant by $81 \%$. Nevertheless, the protection rate was slightly lower than previously reported in a meta-analysis of real-world studies (Kow et al. 2021) conducted before the Delta predominance period; $55 \%$ versus $57 \%$ upon partial vaccination and $81 \%$ versus $88-96 \%$ upon full vaccination.

The reduced effectiveness of the BNT162b2 mRNA vaccine against RT-PCR confirmed COVID-19 caused by the Delta variant relative to other strains of SARS-CoV-2 is most possibly due to the Delta variant notably escapes neutralizing antibodies elicited by vaccination. Previously, in vitro study (Planas et al. 2021) has reported that antibodies elicited by the BNT162b2 mRNA vaccine were efficacious against the Delta variant but about three- to five-fold less potent than they were against the alpha variant (B.1.1.7). It is foreseeable since the BNT162b2 mRNA vaccine encodes an optimized SARS-CoV-2 full-length spike glycoprotein. At the same time, the Delta variant is characterized by the spike glycoprotein mutations T19R, $\Delta 157-158$, L452R, T478K, D614G, P681R, and D950N, which contribute to the regulation of spike glycoprotein dynamics (Kannan et al. 2021). Thus, antibodies elicited by the BNT162b2 mRNA 


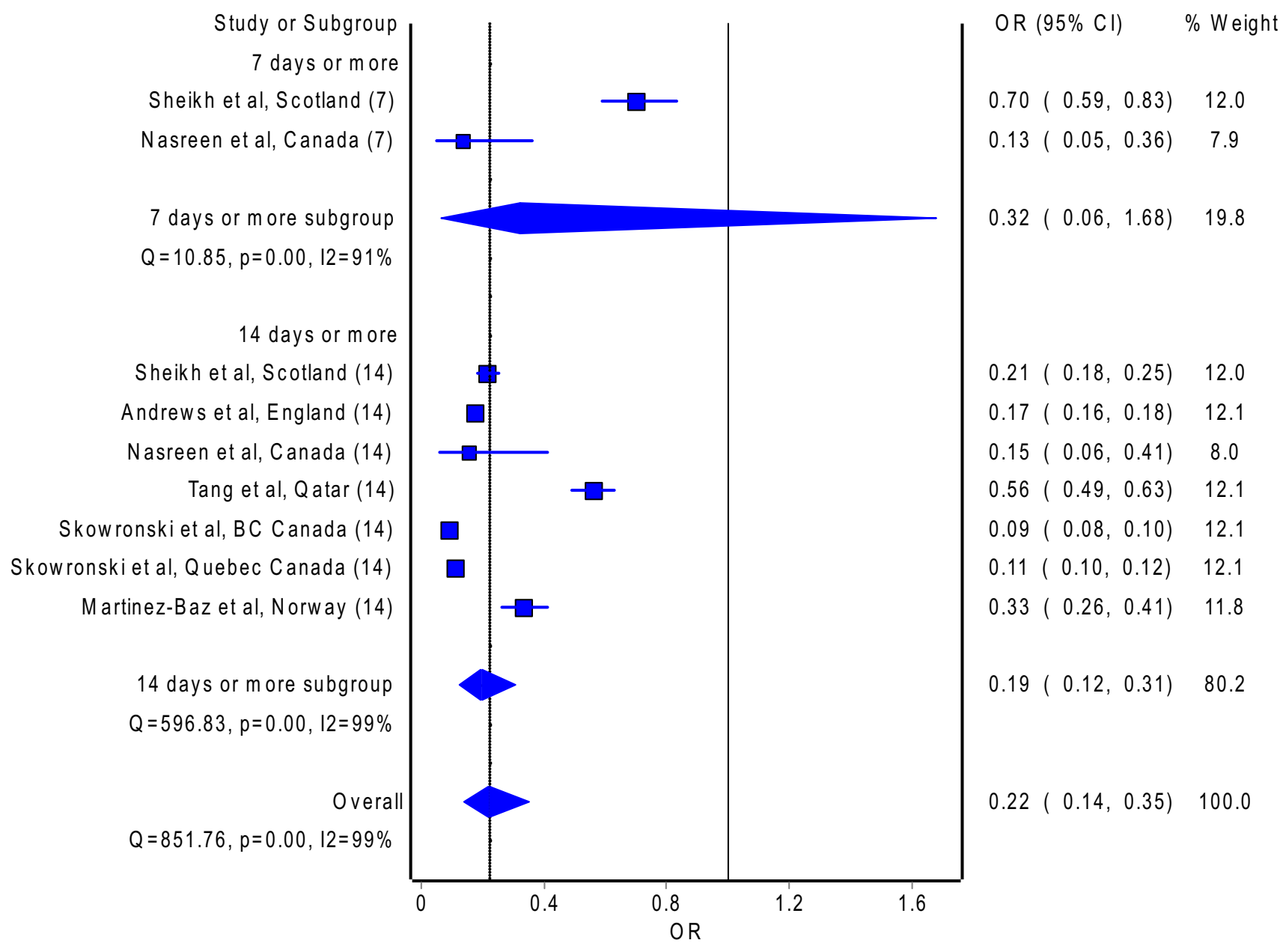

Fig. 3 Pooled odds ratio (OR) of the incidence of COVID-19 7 or 14 days post second dose of vaccine relative to no vaccination

vaccine could have reduced neutralizing effect against the Delta variant.

This systematic review and meta-analysis have its limitations: first, only a small number of studies ( 7 out of 2258 studies screened) were available for inclusion in this systematic review and meta-analysis, and second, all of the included studies in this systematic review and meta-analysis were of the retrospective design, which can have an inferior level of evidence compared with prospective studies. However, we believe it is of utmost importance to disseminate our findings at this stage to alleviate the concerns of practitioners and the general public surrounding the protection rate of the BNT162b2 mRNA vaccine amid the Delta predominance period. In addition, our findings can offer valuable insights to the policy-makers regarding the urgency to administer booster vaccine doses.

In conclusion, the BNT162b2 mRNA vaccine offers a substantial protection rate against RT-PCR confirmed COVID-19 caused by the Delta variant upon full vaccination, albeit with slightly reduced effectiveness relative to other strains of SARS-CoV-2. Therefore, measures should be taken to hasten the global vaccination efforts to curb COVID-19 transmission, which may drive future emergence of variants of concern, and to perform more investigations on the vaccine adjuvants, which can boost longer-lasting immune response upon vaccination. With our current findings and due to emergence of Omicron variant of SARS-CoV-2, we believe that a booster or a third dose of BNT162b2 mRNA vaccine should be considered, and should prioritize those above 65 years old, 18-64 years old with underlying medical condition, and immunocompromised individuals, who are more prone to severe course of COVID-19.

\section{References}

Andrews N, Tessier E, Stowe J, Gower C, Kirsebom F, Simmons R, Gallagher E, Chand M, Brown K, Ladhani S, Ramsay M (2021) Vaccine effectiveness and duration of protection of comirnaty, 
vaxzevria and spikevax against mild and severe COVID-19 in the UK. medRxiv. https://doi.org/10.1101/2021.09.15.21263583

Bian L, Gao Q, Gao F, Wang Q, He Q, Wu X, Mao Q, Liang Z (2021) Impact of the Delta variant on vaccine efficacy and response strategies. Expert Rev Vaccines. https://doi.org/10.1080/14760 584.2021.1976153

Blanquart F, Abad C, Ambroise J, Bernard M, Cosentino G, Giannoli JM, Débarre F (2021) Characterisation of vaccine breakthrough infections of SARS-CoV-2 Delta and Alpha variants and withinhost viral load dynamics in the community, France, June to July 2021. Eurosurveillance 26(37):2100824

Burki TK (2021) Lifting of COVID-19 restrictions in the UK and the Delta variant. Lancet Respir Med 9(8):e85

Kannan SR, Spratt AN, Cohen AR, Naqvi SH, Chand HS, Quinn TP, Lorson CL, Byrareddy SN, Singh K (2021) Evolutionary analysis of the delta and delta plus variants of the SARS-CoV-2 viruses. J Autoimmun 124:102715

Kow CS, Hasan SS (2021) Real-world effectiveness of BNT162b2 mRNA vaccine: a meta-analysis of large observational studies. Inflammopharmacology 29(4):1075-1090

Martínez-Baz I, Trobajo-Sanmartín C, Miqueleiz A, Guevara M, Fernández-Huerta M, Burgui C, Casado I, Portillo ME, Navascués A, Ezpeleta C, Castilla J, Working Group for the Study of COVID19 in Navarre; Investigators, other members of the Working Group for the Study of COVID-19 in Navarre (2021) Product-specific COVID-19 vaccine effectiveness against secondary infection in close contacts, Navarre, Spain, april to august 2021. Euro Surveill 26:2100894

Nasreen S, He S, Chung H, Brown KA, Gubbay JB, Buchan SA, Wilson SE, Sundaram ME, Fell DB, Chen B, Calzavara A (2021) Effectiveness of COVID-19 vaccines against variants of concern, Canada. medRxiv. https://doi.org/10.1101/2021.06.28.21259420

Page MJ, McKenzie JE, Bossuyt PM, Boutron I, Hoffmann TC, Mulrow CD, Shamseer L, Tetzlaff JM, Akl EA, Brennan SE, Chou R, Glanville J, Grimshaw JM, Hróbjartsson A, Lalu MM, Li T, Loder EW, Mayo-Wilson E, McDonald S, McGuinness LA, Stewart LA, Thomas J, Tricco AC, Welch VA, Whiting P, Moher D (2021) The PRISMA 2020 statement: an updated guideline for reporting systematic reviews. BMJ 29:372

Planas D, Veyer D, Baidaliuk A, Staropoli I, Guivel-Benhassine F, Rajah MM, Planchais C, Porrot F, Robillard N, Puech J, Prot M,
Gallais F, Gantner P, Velay A, Le Guen J, Kassis-Chikhani N, Edriss D, Belec L, Seve A, Courtellemont L, Péré H, Hocqueloux L, Fafi-Kremer S, Prazuck T, Mouquet H, Bruel T, SimonLorière E, Rey FA, Schwartz O (2021) Reduced sensitivity of SARS-CoV-2 variant Delta to antibody neutralization. Nature 596(7871):276-280

Sheikh A, McMenamin J, Taylor B, Robertson C, Public Health Scotland and the EAVE II Collaborators (2021) SARS-CoV-2 Delta VOC in Scotland: demographics, risk of hospital admission, and vaccine effectiveness. Lancet 397(10293):2461-2462

Skowronski DM, Setayeshgar S, Febriani Y, Ouakki M, Zou M, Talbot D, Prystajecky N, Tyson JR, Gilca R, Brousseau N, Deceuninck G (2021) Two-dose SARS-CoV-2 vaccine effectiveness with mixed schedules and extended dosing intervals: test-negative design studies from British Columbia and Quebec, Canada. medRxiv. https://doi.org/10.1101/2021.10.26.21265397

Tang P, Hasan MR, Chemaitelly H, Yassine HM, Benslimane F, Al Khatib HA, AlMukdad S, Coyle P, Ayoub HH, Al Kanaani Z, Al Kuwari E (2021) BNT162b2 and mRNA-1273 COVID-19 vaccine effectiveness against the Delta (B. 1.617. 2) variant in Qatar. Nat Med. https://doi.org/10.1038/s41591-021-01583-4

Tartof SY, Slezak JM, Fischer H, Hong V, Ackerson BK, Ranasinghe ON, Frankland TB, Ogun OA, Zamparo JM, Gray S, Valluri SR (2021) Six-month effectiveness of BNT162B2 MRNA COVID19 vaccine in a large US integrated health system: a retrospective cohort study. Lancet 398(10309): 1407-1416

Weinberg GA, Szilagyi PG (2010) Vaccine epidemiology: efficacy, effectiveness, and the translational research roadmap. J Infect Dis 201:1607-1610

Wells G, Shea B, O’Connell D, Peterson J, Welch V, Losos M, Tugwell P (2013) The Newcastle-Ottawa Scale (NOS) for assessing the quality of nonrandomised studies in meta-analyses. http://www. ohri.ca/programs/clinical_epidemiology/oxford.asp. Accessed 28 Sep 2021

Publisher's Note Springer Nature remains neutral with regard to jurisdictional claims in published maps and institutional affiliations. 